\title{
Family Caregiver Perspectives on End-of-Life Dreams and Visions during Bereavement: A Mixed Methods Approach
}

\author{
Pei C. Grant, PhD, ${ }^{1}$ Rachel M. Depner, MS, PhD (C), ${ }^{1,2}$ Kathryn Levy, MSW, AdvStat, ${ }^{1}$ \\ Sarah M. LaFever, PhD, ${ }^{3}$ Kelly E. Tenzek, $\mathrm{PhD},{ }^{4}$ Scott T. Wright, $\mathrm{MS},{ }^{5}$ and Christopher W. Kerr, MD, $\mathrm{PhD}^{1}$
}

\begin{abstract}
Background: End-of-life dreams and visions (ELDVs) are prevalent experiences that provide comfort and meaning to dying individuals. Limited research has examined the impact of ELDVs on the bereaved.

Objective: This study aimed to explore differences in self-reported grief for people whose loved ones shared ELDVs and those who did not, and to describe the role of ELDVs in the grieving process.

Design: Mixed-methods cross-sectional survey.

Settings/Subjects: A total of 228 bereaved family caregivers (FCGs) of patients who died while under the care of a comprehensive hospice program were recruited.

Measurements: Demographics and ELDV prevalence were collected. Bereavement was assessed using the Core Bereavement Items (CBI) a validated measure. Impact on grief was also evaluated using an ad hoc tool. Results: Comfort from dreams significantly related to total CBI score $(r=0.224, p=0.047)$ as well as the images and thoughts $(r=0.258, p=0.025)$ and acute separation subscales $(r=0.224, p=0.047)$. Comfort from dreams had a positive relationship with accepting the reality of loss $(r=-0.511, p<0.001)$, working through the pain of grief $(r=-0.556, p<0.001)$, adjusting to the new environment $(r=-0.405, p=0.001)$, and continuing bonds $(r=-0.538, p<0.001)$. CBI scores were not significantly different between caregivers who reported loved ones with ELDVs and others. Open-ended responses were thematically analyzed resulting in three emergent themes: comfort, reflection and emotions, and sense-making.

Conclusions: ELDVs' impact extends beyond those experiencing them to bereaved loved ones. Bereaved FCGs report that comforting ELDVs experienced by their dying loved ones influenced their grief process in terms of the Worden's tasks of mourning.
\end{abstract}

Keywords: bereavement; end-of-life dreams and visions; family caregivers; hospice care

\section{Introduction}

D REAMS AND VISIONS experienced by the seriously ill have historically been a topic of fascination, and have been observed and documented throughout different cultures. ${ }^{1,2}$ End-of-life dreams and visions (ELDVs) are psychological phenomena that manifest while asleep (dreams) or awake (visions), and typically feature deceased family and friends in the months to hours before death. ${ }^{1,3,4}$ Although ELDVs are common, they have only recently been subjected to scientific inquiry. ${ }^{1,3-9}$ The majority of research focuses on secondary accounts from family caregivers (FCGs) and clinicians, $5,7,8,10$ with few studies soliciting patient perspectives. ${ }^{3,4,11}$ It has been theorized that ELDVs may be inherent to the dying process ${ }^{5}$ serving to help individuals prepare psychologically and/or spiritually for impending death, and may be an

${ }^{1}$ Palliative Care Institute-Department of Research, Center for Hospice and Palliative Care, Cheektowaga, New York.

${ }^{2}$ Department of Counseling, School and Educational Psychology, University at Buffalo, The State University of New York, Buffalo, New York.

${ }^{3}$ VA Western New York Healthcare System, Buffalo, New York.

${ }^{4}$ Department of Communication, University at Buffalo, The State University of New York, Buffalo, New York.

${ }^{5}$ Division of Counseling Psychology, University at Albany, State University of New York, Albany, New York.

Accepted June 11, 2019. 
effective platform for exploring and addressing existential distress and concerns. ${ }^{12,13}$ ELDVs are often significant and meaningful to both dying individuals and observers, and should, therefore, be viewed as valuable opportunities for both clinicians and family to connect with dying individuals.

ELDVs may be mistaken for hallucinations or confused states such as delirium. ${ }^{3,14}$ However, evidence suggests that these experiences are qualitatively different. Although ELDVs are marked by a sense of realism, peace, comfort, organized thinking, and heightened clarity, and can convey profound meaning, $3,4,11,15$ delirium is characterized by disorganized thinking, altered sensorium, agitation, anxiety, or fearfulness. ${ }^{16}$ This is an important distinction, as medical professionals may unnecessarily medicate ELDVs and prevent patients from experiencing potentially meaningful and comforting experiences.

At the bedside, loved ones regard ELDVs as "fascinating, puzzling, but most importantly, a source of personal and spiritual solace." 6 To date, limited research has considered how ELDVs affect bereaved FCGs. ${ }^{6,7,9}$ A recent study in Japan examined the impact of ELDVs on depression in bereaved FCGs and the quality of death of the patient. ${ }^{10}$ With the growing body of literature describing the evident meaning and value inherent in ELDVs, illuminating how ELDVs may affect others beyond the dreamer such as caregivers, can further expound the value of these experiences. Moreover, evaluating bereavement experiences of FCGs can further enhance understanding of ELDVs within a clinical framework.

Several major theoretical perspectives have attempted to describe and explain the complexities of human grief. One such theory, Worden's tasks of mourning, takes a multidimensional flexible active approach aimed toward supporting and empowering bereaved individuals. ${ }^{17}$ This model emphasizes an active process of working through several "tasks" necessary to resolving grief: (1) accepting the reality of loss, (2) processing the pain of grief, (3) adjusting to a world without the deceased, and (4) finding an enduring connection with the deceased while embarking on a new life. Self-assessment of these tasks can be a useful clinical tool to help understand an individual's perspective of their bereavement process. ${ }^{18}$

The aims of this study are (1) to explore differences in grief experiences between those whose loved ones shared ELDVs and those who did not and (2) to describe ELDVs' role in the grieving process of bereaved caregivers.

\section{Materials and Methods}

This is a mixed method cross-sectional study of bereaved caregivers, and was approved by the Social and Behavioral Research Institutional Review Board of a midsize public Northeastern university (FWA00008824), April 10, 2014.

\section{Participants}

Participants were bereaved FCGs of patients who died in August 2012 to September 2013 while enrolled in a comprehensive hospice program. Inclusion criteria were (1) identification as primary caregivers, (2) bereaved at least six months, (3) willingness to be contacted after the death of their loved one, and (4) age 18 years or more. A total of 1600 primary caregivers who met inclusion criteria received a survey by mail. Potential participants were made aware that the completion and return of the questionnaire were regarded as consent to participate in the study, and families who did not want to participate, need not returned the survey.

\section{Survey materials and measures}

Demographics. Caregivers reported age, gender, race, marital status, religious affiliation, relationship to the deceased, and date of death of the deceased.

Core bereavement items. The CBI is a validated tool measuring intensity and severity of bereavement and has been utilized with a variety of individuals including spouses, adult children, and parents. ${ }^{19,20}$ The measure includes 17 items on a 4-point Likert scale (where 0 represents never and 3 represents a lot of the time, always, or continuously). In addition to an overall CBI score, there are also three subscales: images and thoughts (e.g., Do you find yourself preoccupied with images and memories of $X$ ?), acute separation (e.g., Do you find yourself pining for/yearning for $X$ ?), and grief (e.g., Do reminders of $X$ such as photos, situations, music, places, etc. cause you to cry about $X$ ?). Psychometric evaluations of the CBI have found the measure to be reliable with strong internal consistency and concurrent validity.

Dreams/visions prevalence and characteristics. The survey developed for this study was based on previous research, ${ }^{3,4}$ as no standardized tools exist to measure ELDVs. The tool asked participants whether their loved one experienced ELDVs, type of ELDV(s) (awake, asleep, or both), and degree of perceived comfort provided by the experience (rated on a 5-point Likert scale ranging from "strongly agree" to "strongly disagree"). Caregivers were also asked to provide a brief description of the $\operatorname{ELDV}(\mathrm{s})$.

Impact of dreams/visions on grief. There were no specific tools available to evaluate bereaved experiences and grief processing of FCGs. A measure was created based on Worden's task of mourning, ${ }^{17}$ where each question asked about how their loved one's dreams impacted the overall grieving process or a specific task of mourning. Answers corresponded with a 5-point Likert scale ranging from "not at all" to "a great deal" with space to further elaborate. Questions posed on the four tasks of mourning and the overall grieving process are given in Table 1.

\section{Data analysis}

Quantitative analysis. Data were analyzed using SPSS Version 25. Participants were excluded from analyses if they did not complete the measure or answer two or more CBI questions $(n=8)$. Remaining missing data on CBI were imputed using mean imputation. Frequencies for relevant presence, type of ELDVs, and relationship with tasks of mourning were obtained. Differences between demographic groups were analyzed using Kruskal-Wallis and MannWhitney testing. The relationship between bereavement and ELDV status was examined using one-way MANOVA; correlation and $t$-test analyses were used to examine relationships between bereavement selected demographic variables, comfort, and Worden's tasks. 
Table 1. Questions on Participant-Perceived ImPaCt of END-OF-LifE DREAMS AND Visions ON GRIEF

\begin{tabular}{cc}
\hline \multicolumn{1}{c}{ Questions } & $\begin{array}{c}\text { Worden's } \\
\text { task }\end{array}$ \\
\hline 1 To what extent did your loved one's dreams/ \\
visions impact your overall grieving \\
process with the loss of your loved one?
\end{tabular}

${ }^{\text {a }}$ Participants were asked to rate on a 5-point Likert scale: 1-5 ranging from "Not at all" to "A great deal." Participants were also asked to elaborate answers in open-ended format. Questions were adapted from Worden's tasks of mourning. ${ }^{17}$

Qualitative analysis. All open-ended text responses were analyzed using thematic analysis by two researchers. ${ }^{21}$ After familiarization of the data, initial codes were generated, followed by organization into codes. Coded data were then gathered into themes and subthemes, reviewed, and defined iteratively. A report was produced, containing selected exemplary extracts of data that support findings, and relating analyses to the research question as well as literature.

\section{Results}

\section{Demographic data}

A total of 228 surveys were completed and returned. Excluding 30 undeliverable letters, the response rate was $14.5 \%$. The majority of participants identified as white/Caucasian (95.1\%), female (71.4\%), and Catholic (55.4\%), with average age of 64.85 years $(\mathrm{SD}=12.9)$. Most respondents reported losing a spouse $(54.8 \%)$, that their loved one's primary diagnosis was cancer $(61.8 \%)$, and that their loved one passed away within the past $12-24$ months $(59.6 \%)$. Table 2 gives full demographic information.

\section{Quantitative results}

Demographics and core bereavement items subscales. Small but statistically significant positive relationships between age and images and thoughts $(r=0.187$, $p=0.005)$, acute separation $(r=0.146, p=0.031)$, and total core bereavement $(r=0.169, p=0.013)$ were found. Significant differences between CBI subscales and relationship with the deceased existed between groups for all scores $(p<0.001)$. Participants reporting loss of a spouse had significantly higher scores on all subscales than participants who lost a parent $(p<0.001)$. Testing of gender and core bereavement items found no significant relationship between variables. There were no relationships between core bereavement items and race/ethnicity, religion, primary diagnosis of the deceased, or time since death.
Table 2. Participant Demographic Data $(N=228)$

\begin{tabular}{|c|c|c|c|}
\hline Characteristic & Group & $M$ & $S D$ \\
\hline Age (years) & & $\begin{array}{c}64.85 \\
N\end{array}$ & $\begin{array}{c}12.9 \\
\%\end{array}$ \\
\hline \multicolumn{4}{|l|}{ Gender } \\
\hline & Female & 152 & 71.4 \\
\hline & Male & 61 & 28.6 \\
\hline \multicolumn{4}{|l|}{ Race/ethnicity } \\
\hline & White/Caucasian & 214 & 95.1 \\
\hline & Black/African American & 5 & 2.2 \\
\hline & Other & 6 & 2.7 \\
\hline \multicolumn{4}{|l|}{ Religion } \\
\hline & Catholic & 124 & 55.4 \\
\hline & Christian & 78 & 34.8 \\
\hline & None & 16 & 7.1 \\
\hline & Other & 6 & 2.6 \\
\hline \multicolumn{4}{|c|}{ Relationship of deceased with participant } \\
\hline & Spouse & 125 & 54.8 \\
\hline & Parent & 82 & 36 \\
\hline & Sibling & 7 & 3.1 \\
\hline & Other & 14 & 6.1 \\
\hline \multicolumn{4}{|c|}{ Primary diagnosis of loved one } \\
\hline & COPD & 17 & 7.6 \\
\hline & $\mathrm{CHF}$ & 20 & 8.9 \\
\hline & Cancer & 139 & 61.8 \\
\hline & Other & 34 & 15.1 \\
\hline & Multiple diagnoses specified & 15 & 6.7 \\
\hline \multicolumn{4}{|c|}{ Time since loved one's death } \\
\hline & Less than one year & 82 & 39.4 \\
\hline & One to less than two years & 124 & 59.6 \\
\hline & More than two years & 2 & 1 \\
\hline
\end{tabular}

CHF, congestive heart failure; COPD, chronic obstructive pulmonary disease.

ELDV prevalence and characteristics. A total of $27.2 \%(n=62)$ of respondents indicated that their loved ones experienced an ELDV, $18.4 \%(n=42)$ reported no ELDV experience, and the remaining $54.4 \%(n=124)$ were either unsure $(n=118)$ or misunderstood the question $(n=6)$ (e.g., responded about their loved one's hopes and dreams vs. ELDVs). Of the reported ELDVs, 29\% occurred while awake, $22.6 \%$ occurred while asleep, and $48.4 \%$ occurred while both awake and sleep. Nearly half (47.5\%) believed these ELDVs brought comfort to their loved one (agree or strongly agree).

ELDV status and core bereavement. Cronbach's alpha reliability estimates were 0.95 for CBI total, 0.87 for images and thoughts, 0.86 for separation, and 0.92 for grief, indicating good internal consistency. There were no differences in CBI scores (total and subscales) among those whose loved one's experienced an ELDV, did not, or did not know, with no statistically significant difference in CBI scores based on ELDV status, $F(6,432)=0.521, p=0.792$; Pillai's trace $=$ 0.014 , partial $\eta^{2}=0.01$.

ELDV experiences and perceived impact on tasks of mourning. Participants were asked how ELDVs impacted their personal grief experience on each task of mourning. ${ }^{17} \mathrm{~A}$ reliability analysis of this measure yielded a Cronbach's alpha of 0.897, which is above acceptable psychometric thresholds. 
Table 3. Pearson's Correlation Matrix of END-OF-LifE DREAMS AND Vision COMFORT and Core Bereavement Instrument Subscales

\begin{tabular}{lc}
\hline Variable & Comfort \\
\hline Comfort & $-\overline{-}$ \\
Images and thoughts & $0.259^{*}$ \\
Acute separation & $0.224^{*}$ \\
Grief & 0.184 \\
Total CBI & $0.224^{*}$ \\
\hline
\end{tabular}

$* p<0.05$

$\mathrm{CBI}$, core bereavement instrument.

Of those whose loved ones reported ELDVs, more than half $(58.2 \%)$ reported they helped with overall grief (rating as moderate, quite a bit, or a great deal). Almost half (49.3\%) said they helped with accepting the reality of their loss, $46.1 \%$ said that they helped them work through the pain of their grief, 39\% said that they helped them adjust to their new world without the deceased, and $45.9 \%$ believed ELDVs helped maintain their enduring connection with their loved one.

Comfort of ELDV experiences and core bereavement. Correlations between ELDV characteristics and core bereavement subscales showed statistically significant negative relationships between perceived comfort and images and thoughts $(r=0.224, p=0.047)$, acute separation $(r=0.259, p=0.025)$, and total core bereavement $(r=0.224$, $p=0.047)$. The more comforting participants felt the ELDV experience was to their loved one, the lower their subscale scores. Table 3 reports correlation values with significance at 0.05 and 0.005 levels.

Comfort of ELDV experiences and perceived impact on tasks of mourning. There were significant positive correlations between perceived comfort and accepting the reality of loss $(r=-0.551, p<0.001)$, working through the pain of grief $(r=-0.556, p<0.001)$, adjusting to the new environment $(r=-0.405, p=0.001)$, and remaining connected to their loved one $(r=-0.538, p<0.001)$. The more comforting participants perceived an ELDV to be, the more they reported ELDVs helped in working through the tasks of mourning. Table 4 reports Pearson correlation values with significance at 0.05 and 0.005 levels.

Table 4. Pearson's Correlation Matrix of END-OF-LifE DREAMS AND Vision COMFORT AND WORDEN'S TASKS

\begin{tabular}{ll}
\hline Variable & Comfort \\
\hline Comfort & $-\overline{196}$ \\
Overall grieving process & $-0.511^{* *}$ \\
Accepting the reality of the loss (task 1) & $-0.556^{* *}$ \\
Working through the pain of grief (task 2) & $-0.405^{* *}$ \\
Adjusting to an environment without their & \\
$\quad$ loved one (task 3) & $-0.538^{* *}$ \\
Remaining connected with their loved one & \\
$\quad($ task 4) & \\
$* * * p<0.005$. &
\end{tabular}

\section{Qualitative results}

Three themes emerged from bereaved caregivers' openended responses: comfort, reflection and emotions, and sense-making (Table 5).

Comfort. Participants commonly noted comfort provided by ELDVs describing solace, peace, or reassurance. Often, "comfort" was explicitly mentioned. One participant noted, "He did find comfort talking to and seeing people who passed before him." Comfort for the caregiver also emerged as a subtheme. For example, "When he told me that he saw his favorite sister [deceased] hold out her hands to him, it made me feel comforted because I knew it comforted him."

Reflection and emotions. Upon reflecting about ELDV experiences, a variety of feelings and emotions emerged, including positive, negative, or mixed/contrasting. The majority of emotions were traditionally positive. "I was happy to think our loved ones had come to make his passing easy" or "I thought he was happy, it gave me peace." A few described ELDVs or their reaction as negative. One said, "The fact that he was fearful of some things troubled me. Not knowing if he came to peace with these fears bothers me at times." A handful of participants expressed mixed feelings,

TAble 5. List of Themes And Subthemes

Theme Subtheme

Comfort

Comfort for patient

ELDVs provided feelings of consolation, reassurance, or solace to the dreamer (patient)

Comfort for caregiver

ELDVs directly provided comfort (consolation, reassurance, or solace) to the caregiver, or the mere observation of the ELDV being comforting to the patient can result in comfort for caregiver before or during bereavement process

Reflection Positive

Upon reflection of the ELDV experience, feelings and emotions were affirmative and favorable

Negative

Feelings and emotions upon reflection were distressing, disconcerting, troubling, regretful, and unfavorable

Mixed/contrasting

Caregiver experience feelings that were contradictory or conflictual

Sense- Religious/spiritual process

making Caregiver made sense of ELDVs to be transcendent experiences with a higher being(s) or higher state

Natural dying process Caregiver's understanding of ELDVs was part of progression or trajectory of dying

Medical attribution

Caregiver attributed ELDVs to be part of side effect of drugs, impairments from serious illness, or cognitive decline

ELDVs, end-of-life dreams and visions. 
with a juxtaposition of positive (comfort/happiness) and negative feelings (sadness), largely because of recognition that death was near "I was glad he saw his brother, but did not want him to go with him."

Sense-making. In sharing ELDV experiences, the theme of sense-making emerged, including responses describing how caregivers tried to understand, explain, or conceptualize ELDVs and incorporate them into their own worldview. The subtheme of Religious/Spiritual Process was the most apparent. Many felt that ELDVs were an indication that their loved one was "going to a better place." The involvement of "God" and other religious figures was also common. One respondent said, "It gave me great comfort to know that angels were waiting for him to escort him to heaven." Others considered ELDVs to be medically related, due to either cognitive decline or medication: "It was the pain medication alone causing these visions and had no more significance other than being a side effect of the drug's affecting her brain chemically."

\section{Discussion}

This study explored relationships between ELDVs and bereavement from the perspective of FCGs. There is little literature on the effects of ELDVs on FCG grief outcomes, although supporting literature indicates great comfort to individuals having ELDVs. ${ }^{1,3-9}$ To our knowledge, this is the first study examining the relationship between the grieving process and exposure to a dying loved one's ELDVs using a mixed-methods approach.

The main finding of this study is that bereaved caregivers who reported that their deceased loved ones' ELDVs appeared comforting also reported significant positive effects on their grief process. This study extends what is known about the relationship between ELDVs and the impact of comfort beyond the dying individual to loved ones. ${ }^{3}$ The more comforting caregivers perceived ELDVs to be for their loved one, the better their overall CBI scores. This was also true for CBI subscales of images and thoughts and acute separation. Likewise, there was an association between perceived ELDV comfort and working through the grief process. Perceived ELDV comfort and its role in bereavement are further substantiated by the qualitative findings, and that comfort for the dying patient can translate to comfort for caregivers.

Positive reflections on ELDVs allowed caregivers to construct and make sense of ELDV experiences in a meaningful way. Even those who experienced mixed or contrasting feelings were able to make meaning. Sense-making was often through religious and spiritual lenses, wherein belief in an afterlife, God, angels, or heaven helped caregivers through their grief experience. Furthermore, faith that their loved one will not be alone in afterlife and hope of being reunited allows caregivers to adjust to a world without the loved one, while maintaining a continuing bond.

Participants who reflected on ELDVs negatively were typically caregivers whose loved one had distressing dreams/ visions. ELDVs are not strictly comforting and can elicit a range of emotions, including those associated with distress. ${ }^{3,4}$ In this study, distressing dreams left caregivers uneasy, often attributing the dreams to medication side effects or cognitive impairment. It is possible that caregivers confused ELDVs with delirium. Others who reflected negatively focused on their personal reaction to the experience, expressing regret at not validating their loved one's experience or remorse for not taking the opportunity to ask more questions.

The prevalence of ELDVs reported in this study (28\%) is comparable with that reported in previous studies involving secondary accounts. ${ }^{7,10,15}$ However, over half of the respondents indicated that they were unsure whether there were any ELDVs, suggesting a lack of awareness or understanding of this phenomenon. This difficulty poses a barrier to accurately evaluating the impact of ELDVs on bereavement. Owing to the salience of ELDVs to both the dying individual and their families, it may be helpful for discourse and openness to be facilitated by clinical staff.

\section{Limitations}

This study included several limitations. First, previous research has demonstrated that there is general lack of awareness, understanding, and education surrounding ELDVs, $, 2,6,8,15$ and thus participant interpretation of ELDVs may lead to misperceived survey responses, or lack of response. An operationalized definition distinguishing ELDVs from phenomenologically similar experiences such as delirium should be used in future studies. Second, this study asked FCGs retrospectively about their loved one's experiences and consequently results are subject to recall bias. Third, lack of psychometrically validated instruments on this topic limited validity of findings. Future research should address this. Fourth, no significant findings were identified in overall CBI and subscale scores between caregiver groups. Grief is a multifaceted process influenced by many factors, ${ }^{22}$ and the personal and varied aspects such as encountering ELDVs need to be further studied. Future studies should look at other factors, such as openness to ELDVs, which may influence grief outcomes. Lastly, there were a couple of limitations to the generalizability of the findings, specifically the homogeneity of the sample and the low survey response rate.

\section{Conclusions}

This study is the first to suggest that comfort from ELDVs experienced by the dying can positively influence grief experiences of the bereaved. The importance of ELDVs extends beyond that of the persons having the experience to their loved ones. For this reason, FCGs, alongside clinicians, should be educated on how to distinguish between delirium and ELDVs. A greater understanding and acknowledgment of ELDVs are necessary within the realms of death, dying, and bereavement to improve patient care and clinical outcomes. Future research should aim to further expand the understanding of the impact of ELDVs beyond the dying individual.

\section{Acknowledgments}

The authors express gratitude to the families who took time to share their experiences in the midst of their grief journey. Furthermore, we are thankful to the PCI research volunteers and interns who helped with survey mailing preparation and data entry. We are also grateful to Debra Luczkiewicz, MD, and David Byrwa, MS, for their editorial comments. Lastly, 
the authors thank the Hospice Foundation of Western New York and their donors for their ongoing support.

\section{Author Disclosure Statement}

No competing financial interests exist.

\section{References}

1. Mazzarino-Willett A: Deathbed phenomena: Its role in peaceful death and terminal restlessness. Am J Hosp Palliat Med 2009;27:127-133.

2. Fenwick P, Lovelace H, Brayne S: End of life experiences and their implications for palliative care. Int J Environ Stud 2007;64:315-323.

3. Kerr CW, Donnelly JP, Wright ST, et al.: End-of-life dreams and visions: A longitudinal study of hospice patients' experiences. J Palliat Med 2014;17:296-303.

4. Nosek CL, Kerr CW, Woodworth J, et al.: End-of-life dreams and visions: A qualitative perspective from hospice patients. Am J Hosp Palliat Med 2015;32:269-274.

5. Brayne S, Farnham C, Fenwick P: Deathbed phenomena and their effect on a palliative care team: A pilot study. Am J Hosp Palliat Med 2006;23:17-24.

6. Fenwick P, Brayne S: End-of-life experiences: Reaching out for compassion, communication, and connection-meaning of deathbed visions and coincidences. Am J Hosp Palliat Med 2011. DOI:10.1177/1049909110374301

7. Kellehear A, Pogonet V, Mindruta-Stratan R, Gorelco V: Deathbed visions from the republic of Moldova: A content analysis of family observations. Omega (Westport) 20112012;64:303-317.

8. Lawrence M, Repede E: The incidence of deathbed communications and their impact on the dying process. Am J Hosp Palliat Med 2012;30:632-639.

9. Muthumana SP, Kumari M, Kellehear A, et al.: Deathbed visions from India: A study of family observations in Northern Kerala. Omega (Westport)2010-2011;62:91-109.

10. Morita T, Naito AS, Aoyama M, et al.: Nationwide Japanese survey about deathbed visions: "My Deceased Mother Took Me to Heaven.” J Pain Symptom Manage 2016;52: 646-654.e5.

11. Dam A: Significance of end-of-life dreams and visions experienced by the terminally ill in rural and Urban India. Indian J Palliat Care 2016;22:130-134.
12. Goelitz A: Exploring dream work at end of life. Dreaming 2007;17:159-171.

13. Wright ST, Grant PC, Depner RM, et al.: Meaningcentered dream work with hospice patients: A pilot study. Palliat Support Care 2015. DOI:10.1017/S147895151 4001072.

14. Betty LS: Are they hallucinations or are they real? The spirituality of deathbed and near-death visions. Omega $\mathbf{J}$ Death Dying 2006;53:37-49.

15. Fenwick P, Lovelace H, Brayne S: Comfort for the dying: Five year retrospective and one year prospective studies of end of life experiences. Arch Gerontol Geriatr 2010;51: 173-179.

16. American Psychiatric Association: Diagnostic and Statistical Manual of Mental Disorders DMS V, 2013. DOI: 10.1176/appi.books.9780890425596.744053

17. Worden JW: Grief Counseling and Grief Therapy, 3rd ed. Springer Publishing Company, 2008. https://books.google .com/books?id=Dz6mti2YLy4C

18. Neimeyer RA: Techniques of Grief Therapy: Assessment and Intervention. Taylor \& Francis, 2015. https://books.google .com/books?id=c4n4CgAAQBAJ

19. Burnett P, Middleton W, Raphael B, Martinek N: Measuring core bereavement phenomena. Psychol Med 1997; 27:49-57.

20. Holland JM, Nam I, Neimeyer RA: A psychometric evaluation of the core bereavement items. Assessment 2012;20: 119-122.

21. Braun V, Clarke V: Using thematic analysis in psychology. Qual Res Psychol 2006;3:77-101.

22. Thompson N, Allan J, Carverhill PA, et al.: The case for a sociology of dying, death, and bereavement. Death Stud 2016;40:172-181.

Address correspondence to:

Pei C. Grant, PhD

Department of Research

Center for Hospice and Palliative Care

225 Como Park Boulelvard

Cheektowaga, NY 14227

E-mail:pgrant@palliativecare.org 